\title{
Five Case Studies of Soldiers with Painful Clavicular Fracture Non-Union
}

\author{
Mr A Edwards \\ FRCS \\ Senior Orthopaedic SHO \\ Lt Col F Khan \\ FRCS, RAMC \\ Consultant Orthopaedic Surgeon
}

\author{
Maj AL Smith \\ FRCS, RAMC \\ Specialist Registrar in Orthopaedics
}

MDHU Frimley Park Hospital, Portsmouth Road, Frimley, Surrey GUI6 5UJ

SUMMARY: We report a short case series of 5 servicemen who had sustained closed clavicular fractures, 4 of whom presented to our clinic with a painful non-union, and 1 required primary fixation for tenting of the skin. Our treatment consisted of open reduction and internal fixation of these non-unions. This resulted in all these men returning to duties with painfree shoulder girdles within 6 months. A complication in these cases was one of a prominent plate which rubbed on straps and required removal of the plate after union had occurred resulting once again in a painfree shoulder girdle and a return to full duties. We advocate early orthopaedic referral in cases of painful clavicular non-union and where appropriate, open reduction and internal fixation with the expectation of an early return to full duties with a pain free shoulder girdle.

\section{Introduction}

Extensive clinical studies reported in the literature have indicated that non-operative management is the treatment of choice for clavicular fractures. However, when painful nonunion occurs, open reduction and internal fixation may be appropriate. We report five cases of clavicular fractures in soldiers treated in this way.

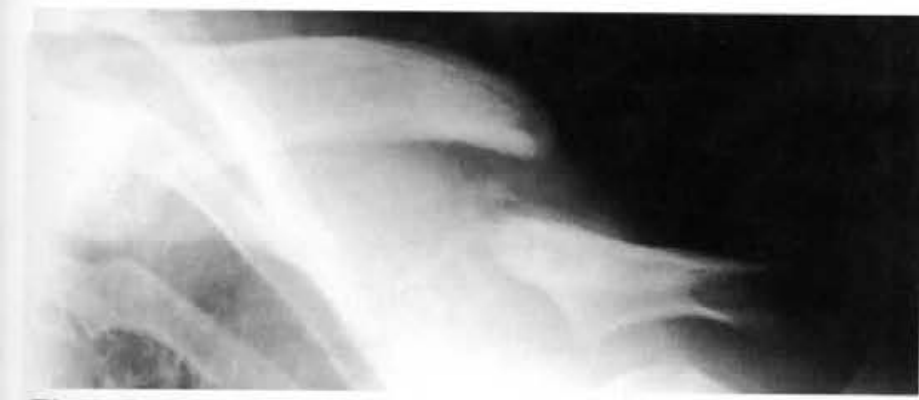

Fig 1. Left clavicular fracture painful non-union 7 months after injury.

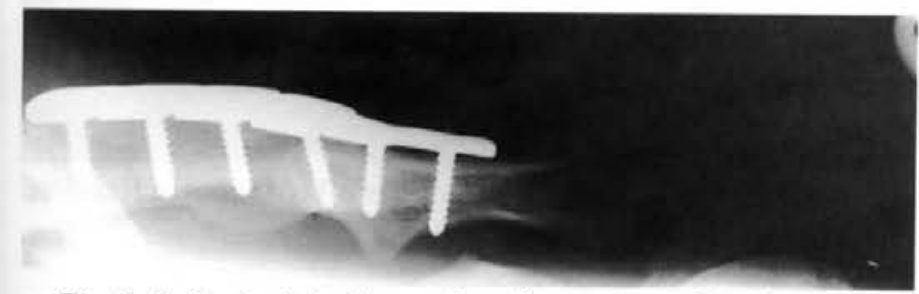

Fig 2. Left clavicle 4 months after open reduction and internal fixation showing good callus formation.

\section{Case Reports}

Case 1 (Figs 1\&2)

This 26 year old Lance Corporal fractured his left clavicle and was treated with a broadarm sling followed by exercises. He presented to clinic six months later complaining of a sharp pain over his left clavicular fracture site. Clinical examination and $\mathrm{X}$-rays confirmed non-union.

He underwent open reduction, iliac crest bone grafting and internal fixation with a 6 hole reconstruction plate. At 4 month follow up X-ray show good callus formation and at 6 months he returned to full duties.

No problems were reported at 16 month follow up.

\section{Case 2 (Figs 3-5)}

A 20 year old Sapper sustained a left clavicular fracture which was treated in a broadarm sling followed by physiotherapy. One year later he presented to our clinic complaining of pain in the shoulder, weakness in lifting and of being unable to carry anything over his shoulder. X-rays confirmed non-union.

He underwent open reduction, iliac crest bone grafting, and

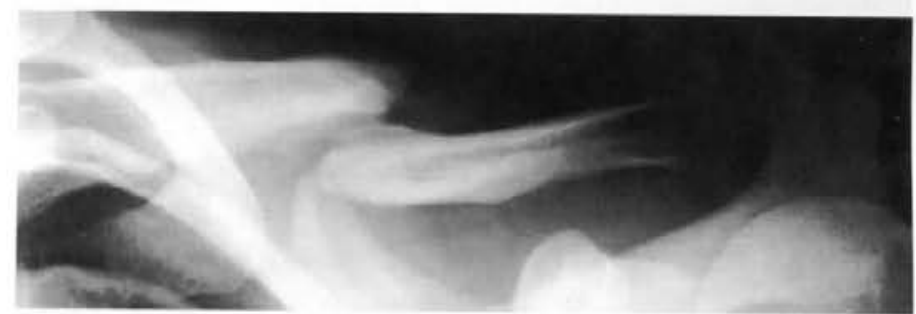

Fig 3. Left clavicle 1 year after fracture.

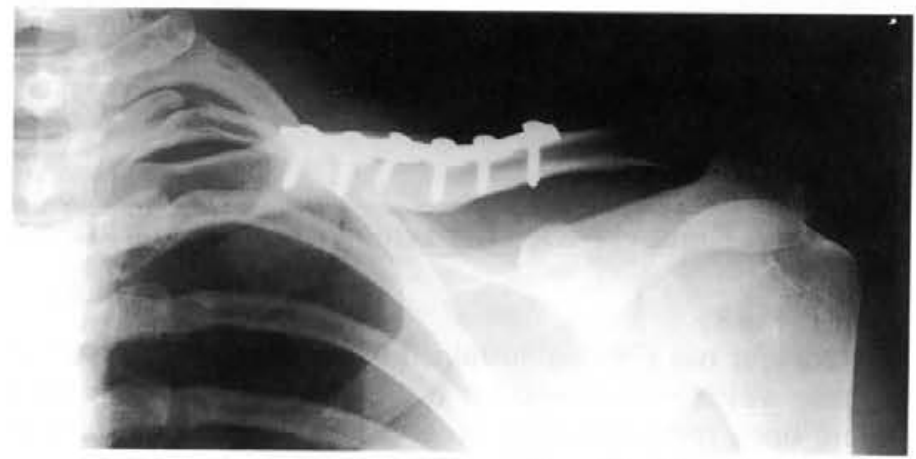

Fig 4. Left clavicle 10 months after fixation. 


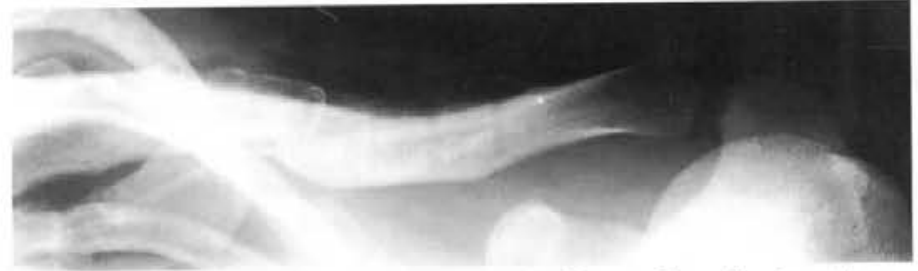

Fig 5. Left clavicle after removal of metalwork showing sound union of the fracture.

internal fixation with a 6 hole contoured reconstruction plate.

At 10 month review the X-ray showed union of the fracture and he had no pain nor weakness of the shoulder. However, the metal work was prominent and was causing irritation when the patient was carrying a rucksack.

He had the plate removed 1 year after the first operation. At 6 month follow up he reported no further problems.

\section{Case 3 (Figs 6\&7)}

This 20 year old private sustained a closed fracture of the right clavicle which was treated primarily by open reduction and internal fixation with an 8 hole plate.

Eighteen months later he presented to our clinic complaining that the prominent clavicular plate was causing irritation when carrying webbing. He had returned to full duties after his primary fixation and reported no other problems.

X-rays showed that the fracture had united under the plate. The metalwork was therefore removed.

He returned to full duties after 10 weeks and at 1 year follow up he had full painfree function.

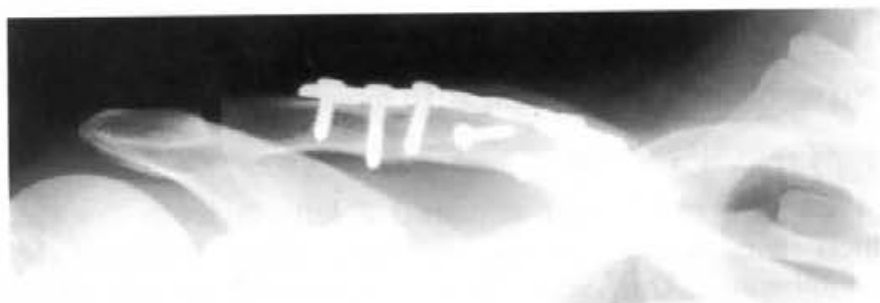

Fig 6. Right clavicle 18 months after fixation.

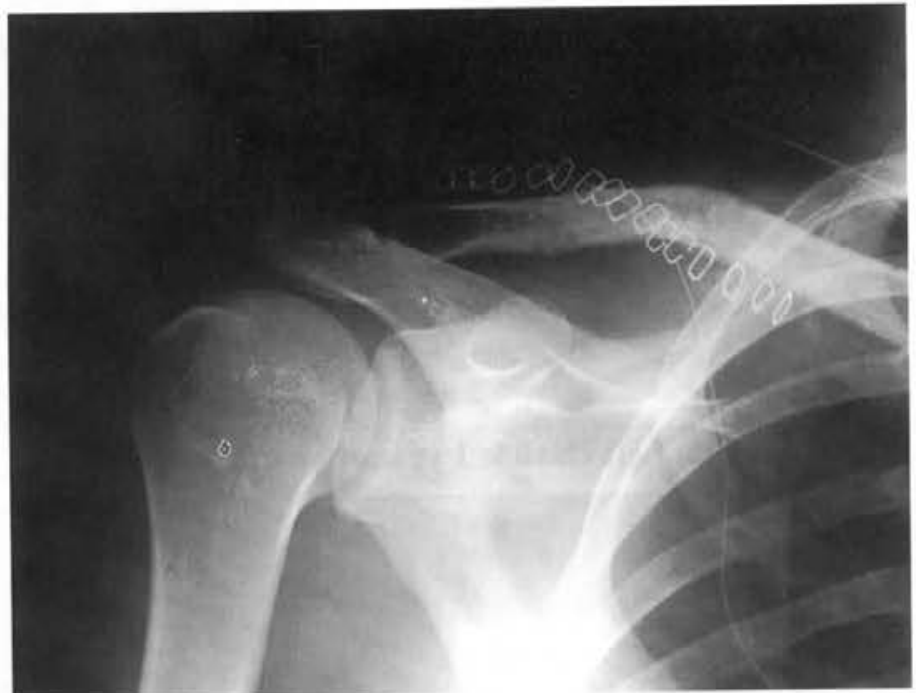

Fig 7. Right clavicle after removal of metalwork.

\section{Case 4 (Fig 8)}

A 26 year old Captain sustained a left clavicular mid-shaft fracture which was treated for 6 weeks in a broad arm sling before open reduction, iliac crest bone grafting, and internal fixation with a 6 hole plate was performed.

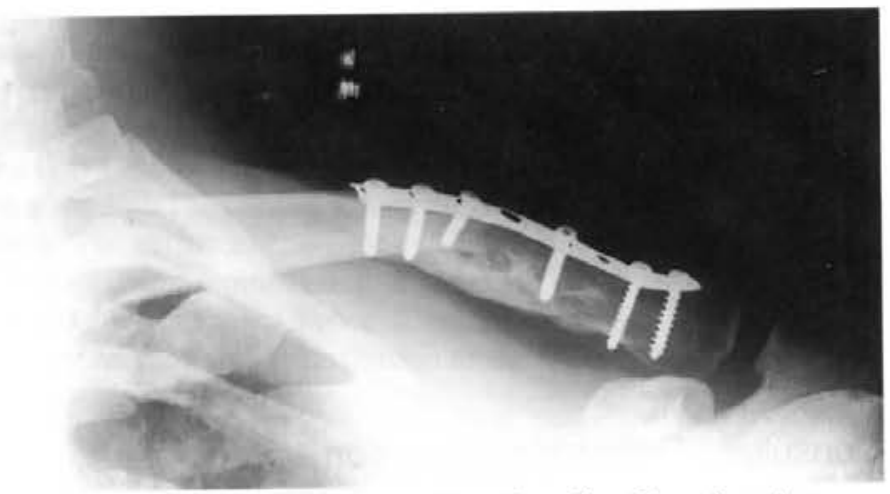

Fig 8. Left clavicle 6 months after fixation showing boney union.

After 6 months there was evidence of bone union and he returned to full duties. However, at 12 months he presented with blistering and pain over the clavicular plate as a result of wearing webbing. The plate was removed under general anaesthesia.

At two year follow up he reported no further problems.

\section{Case 5 (Fig 9)}

A 24 year old male Lance Corporal sustained a mid-shaft left clavicular fracture which was treated with a broad arm sling initially and then with physiotherapy for 4 months.

He reattended clinic complaining of pain, tenderness and a clicking sensation over the left clavicular fracture site. He was unable to wear webbing and felt that he had lost strength in his left arm.

At 7 months post injury he underwent open reduction, iliac crest bone grafting and fixation with a contoured 6 hole reconstruction plate.

He returned to full duties at 6 months and at one year follow up reported no further problems.

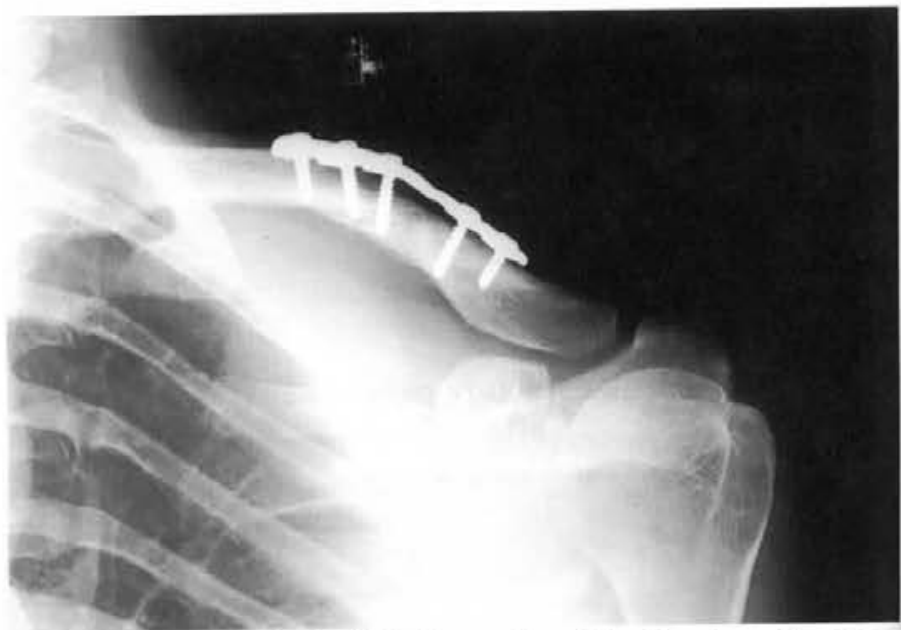

Fig 9. Case 5. Left clavicle 6 months after fracture fixation.

\section{Discussion}

Closed clavicular fractures are invariably treated nonoperatively in a broadarm sling followed by exercises. Malunion is the rule, but in general this causes no functional deficit nor discomfort.

Between 0.9 and $4 \%$ of these injuries progress to non-union. with patients complaining of pain, crepitation, movement at the fracture site and a prominent tender lump (1).

Approximately $85 \%$ of non-unions occur in middle third clavicular fractures, this area being subjected to the greatest torsional loads on the clavicle and being relatively denuded of 
muscle attachments, the poorest blood supply.

Indications for open reduction and internal fixation of clavicular non-unions include a painful non-union that limits function and range of movement, and brachial plexus compression (2).

The AO $3.5 \mathrm{~mm}$ reconstruction plate is preferred at our hospital as the implant of choice. It must be carefully moulded to the shape of the clavicular segment to be fixed. Resection of bone at the site of the non-union is invariably required and interposition autologous cancellous bone graft is used to fill the gap and improve callus formation. Clavicular shortening of over $10 \mathrm{~mm}$ is said to increase the possibility of some abduction weakness, but none of our subjects reported this problem, after plating.

Jupiter and Leffert (3) reported an 85 to $90 \%$ rate of good to excellent results overall in their series of clavicular fractures requiring open reduction and internal fixation. Our small series matches these results.

Finally, clavicular plating may be prominent under the skin and therefore cause irritation or blistering. This is of particular importance to soldiers who carry heavy weights over their shoulders. Removal of the plate after the fracture has united usually results in no further complications.

A painful non-union of a mid-clavicular fracture can be a debilitating problem, especially to servicemen who have a high demand and expectation of their shoulder girdle. Referral to an orthopaedic specialist for assessment and operative treatment should not be delayed, if there is not rapid resolution with conservative treatment.

\section{REFERENCES}

1. RockWOOD, JR CA, GREen DP, Bucholz RW, Fractures in Adults, 3rd ed. Philadelphia: JB Lippincott Co, 1996: 954955, 968.

2. Browner BD, Jupiter JB, Levine AM, Trafton PC Clavicular fractures, in Skeletal Trauma. Phildadelphia: WB Saunders Co, 1992: 1303-1304.

3. Jupiter JB, LeFFERT RD. Non-union of the clavicle: Associated complications and surgical management. J Bone Joint Surg 69-A: 730-760, 1987.

व 